\title{
Control and optical mapping of mechanical transitions in polymer networks and DNA-based soft materials
}

\author{
Giuliano Zanchetta ${ }^{1}$ \\ Dept. of Medical Biotechnology and Translational Medicine, University of Milano, Via \\ Fratelli Cervi 93, 20090 Segrate, Italy
}

\begin{abstract}
Complex mechanical changes in response to an external trigger are pervasive in natural soft materials and often sought for applications. Be it the reversible stiffening of sea cucumber, the failure of a polymeric or colloidal gel under load, or the dissolution of a biosensing hydrogel upon target binding, mechanical transitions are typically enabled, and critically affected, by heterogeneous structures and reversible bonds. New possibilities to monitor evolving properties and to gain access to stress propagation with temporal and spatial resolution are being disclosed by mechanochromic molecules and molecular complexes, which transduce a mechanical stress into a light signal and act as built-in stress reporters. I will review recent strategies and identify future directions for the design of mechanically responsive soft networks and for their optical mapping, focusing particular attention on the emerging class of hydrogels based on DNA self-assembly.
\end{abstract}

Keywords: DNA self-assembly, Hydrogels, Mechanochemistry, Failure, Transient networks, Responsive networks, Yielding, Built-in strain reporters

\section{Introduction}

Nature is a constant source of inspiration and building blocks for the design of functional and adaptive materials, whose properties change in a selective

\footnotetext{
${ }^{1}$ giuliano.zanchetta@unimi.it
} 
manner in response to an external stimulus, such as mechanical stress, heat, 5 light, exposure to water or specific chemicals [1, 2, 3]. Proteins, for example, perform multiple tasks by fine tuning and switching of their internal structures and overall conformation. Efforts to understand how the content of secondary structure affects the force required for unfolding and the degree of deformation before unfolding [4] are guiding the rational design of hydrogels with tailored mechanical properties [5, 6, based or not on peptides. For example titin, one of the main proteins in human muscles and responsible for their passive elasticity, is composed by unstructured segments connecting repeated domains, which reversibly unfold upon stretching and hence absorb energy. For this reason, it is one of the most mimicked design models for adaptive materials, like modular 15 polymers with unfolding cross-linker modules based on Hydrogen bonds [7]. The high rupture force and spontaneous re-folding of each module is translated into a large energy dissipation during plastic deformation, hence leading to materials with high mechanical strength, fracture toughness and elasticity [8]. DNA, a much simpler polymer than proteins in many respects, offers nonetheless a wide variety of possible design and responsive motifs thanks to its modular linear structure and specific recognition, which allowed the explosion of the so-called DNA nanotechnology [9] and the creation of self-assembled, responsive materials [10].

Triggered mechanical changes (like stiffening, gelation, yielding, failure and recovery under external stimuli) are ubiquitous across soft and biological matter and are critical for applications ranging from drug delivery systems to selfhealing coatings to artificial muscles [11. Key to such responses is often a heterogeneous dynamic architecture, in which the association of building blocks can be reversibly switched between distinct states. However, the presence of multiple length and time scales also makes it challenging to understand, predict and control mechanical response, which is often non-homogeneous. Among the various strategies constantly devised to highlight and map global, local and molecular rearrangements inside gels and networks, an emerging and attractive approach is mechanochromism, i.e. a class of methods to transduce stress 
propagation into an optical signal. Mechanochromic molecules and complexes can thus act as built-in reporters of forces or deformations. Again, nature is a constant source of inspiration [3].

The goal of this article is to provide an overview of the most interesting and recent results in the creation of responsive networks and in the optical 40 mapping of mechanical changes like reversible gelation or stress-induced failure, with a special care for soft materials based on nucleic acids. I will first describe the current strategies adopted to control mechanical transitions and response in transient networks, especially as regards DNA-based hydrogels. I will then discuss the main approaches to mechanochromism and molecular switches in 45 polymers and hydrogels and I will give examples of their use to gain spatial and temporal mapping of stress propagation and fluidization. Finally, I will try to suggest directions for the future translation of novel motifs to DNA-hydrogels.

\section{Keep on movin': transient networks}

A particularly attractive architecture to create reconfigurable materials is that of transient networks, i.e. 3D scaffolds based on the association of building blocks like polymers, biological macromolecules or colloidal particles. The network structure assures mechanical robustness, but at the same time allows reversible (dis)assembly and thereby adaptability. A recent breakthrough is represented by polymer vitrimers: their network of covalent links can rearrange its topology via a bond-switching reaction that favours healing and relaxation of internal stresses while preserving the connectivity, so that such materials can be heated and worked to any shape without dissolving [12. However, the most natural choice to obtain rearranging networks is to exploit non-covalent, reversible bonds. Hydrophobic and dipolar interactions are widespread in nature but mainly non-specific, while only few specific, non-covalent interactions can work in aqueous medium. Typical binding motifs, also found in nature and widely exploited for material design, are Hydrogen bonding, multivalent ionic interactions, metal-ligand coordination and host-guest complexation [13, 1]. 

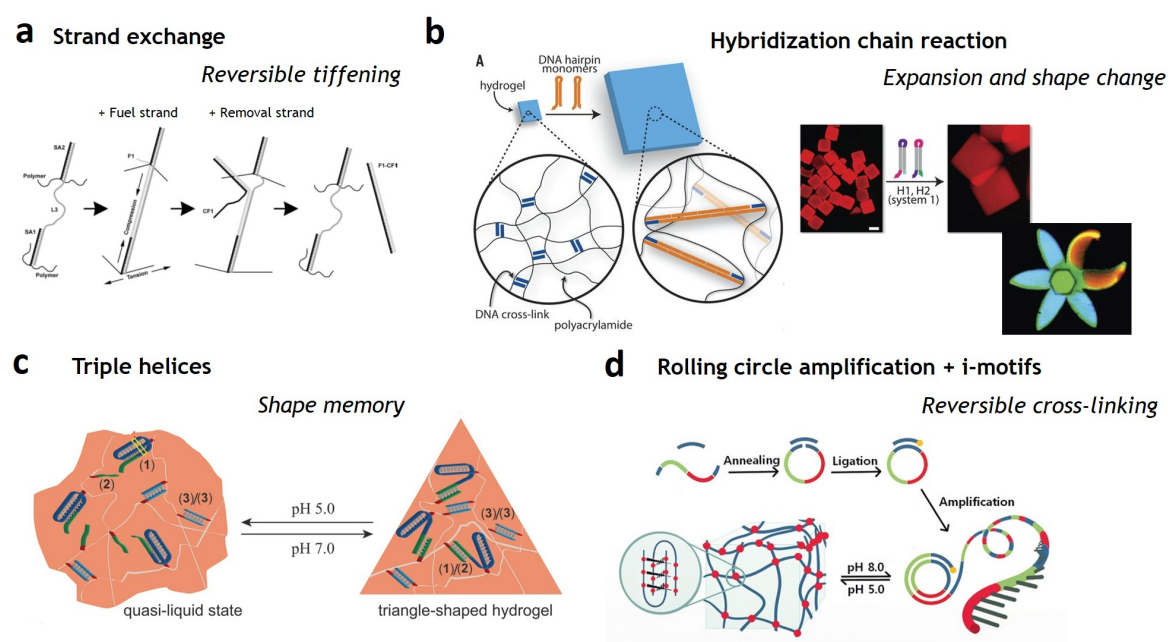

C Triple helices

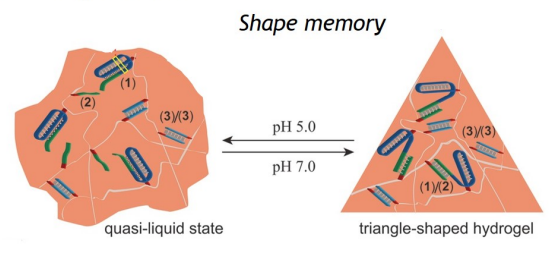

d Rolling circle amplification $+\mathrm{i}$-motifs

Figure 1: Typical DNA motifs used to induce mechanical transitions in hydrogels. (a) Strand exchange of fuel and anti-fuel filaments triggers reversible stiffening. (b) Hybridization chain reaction elongates cross-links and guides the expansion of a hydrogel and its programmable shape change. Scale bars $1 \mathrm{~mm}$. (c) The state of a shape-memory hydrogel is controlled through the $\mathrm{pH}$-triggered competition between duplex and triple helix conformations. (d) An all-DNA hydrogel is produced through Rolling Circle Amplification with i-motifs acting as reversible, pH-dependent cross-links. Reproduced with permission from refs. 14, 15] (AAAS), [16] and [17.

An extensive description of the modes of self-assembly mediated by noncovalent interactions and of the resulting mechanical properties goes beyond the scope of this review. Here, I will discuss in some details only DNA-mediated interactions, then providing specific examples related to mechanochromism in the next section.

\subsection{DNA-based dynamic materials}

In recent years, DNA emerged as a key player in material science [9. Its base sequence encodes structural and functional information into the biopolymer, making it an ideal building block for an incredible set of nano- and microconstructions [9] and for a variety of soft materials [18. Fine tuning of the structure and of the interaction energy can be achieved through proper choice of 
length and sequence of the DNA strands and through control of temperature and ionic strength. Furthermore, isothermal strand displacement is also possible, in which one strand of DNA displaces another in binding to a third strand with partial complementarity to both. A toehold, a short single-stranded overhang region, seeds the strand displacement, thereby tuning kinetics and opening the way to dynamically controlled structures.

Besides Watson-Crick double-stranded helices, formed by complementary base pairings, DNA also features a wide variety of responsive structures [19], such as pH-driven triple helices of C-GC or T-AT, i-motif quadruplexes of hemi-protonated cytosines, also pH-dependent, guanosine quadruplexes bound through Hoogsteen pairing. Furthermore, DNA sequences - and even more so RNA - can be screened and selected for a specific functionality, like binding of a small molecule through folded conformations called aptamers. With this rich toolbox it is possible to design dynamic hydrogels with responsive properties, with applications in biosensing, controlled delivery, cell scaffolding and soft actuation [19, 10]. Two general strategies are adopted: either tethering DNA to a polymer chain (mostly polyacrylamide or peptides), providing it with reversible cross-links and stimuli-responsive elements; or creating DNA-only hydrogels in which nucleic acids also constitute the backbone [10].

DNA-polymer hybrid hydrogels. Through strand exchange reactions, DNA can impart tunable mechanical properties to hydrogels, without changing temperature or buffer composition. In a pioneering demonstration [14] (Fig. 1]), each cross-link contained a flexible, unpaired region to which a DNA strand with a complementary base sequence (fuel strand) could bind, hence changing the stiffness of the cross-links. The gel was restored to its initial nanostructure through a removal strand, complementary to the fuel, which bound to the toehold and removed the fuel. This behaviour can be literally expanded through Hybridization Chain Reaction (HCR), in which the assembly of two different single stranded units, each forming a stable hairpin, is triggered by a third filament, yielding a long double-stranded structure. Hydrogel expansion up to 100 times in volume 
limitation) of only relying on one-pot, hierarchical self-assembly of DNA strand with no need for chemical modifications. The typical approach makes use of structurally well-defined units formed by multiple DNA strands (like for example nanostars with controlled valence), interacting through sticky overhangs to 
form transient networks with tunable lifetime. The exquisite control over structure and interactions enabled the realization of equilibrium gels with Arrhenius temperature-dependence of viscosity over five orders of magnitude 21. and is at the basis of the design of a DNA vitrimer 22]. DNA nanostars phase behaviour, connectivity, and the conditions for the formation of an elastic, stress-bearing network have been widely investigated [23, 24]. Through an active microrheology technique (conceptually as simple as dragging a bead through the sample with a calibrated force and measuring its velocity), the whole temperaturecontrolled transition from a Newtonian dispersion to a power-law fluid to a shear-thinning fluid was explored $[23]^{*}$. The system evolved from a fluid of independent nanostar clusters to a rearranging network of short-lived bonds to a more stable network fractured by the moving bead. The whole behaviour could be described on the basis of the energy of single bonds and of network topology only.

A high degree of programmability and control over mechanical properties could also be obtained through single strands, 36-72 bases in length, with different self-complementary domains. In solution, such domains paired with identical domains in other filaments, resulting in the single-step assembly of a hydrogel with sequence-designed effective valence, thermal stability, pore size and elastic moduli $[25]^{*}$. In an effort to improve the control over the final structure of selfassembled hydrogels, a variation of the linear HCR, clamped HCR, has been recently proposed [26]. One of the HCR hairpins formed self-dimers which protected it against interaction with the second hairpin, unless an initiator strand was introduced. Three-arm and four-arm junctions resulted, and 3D hydrogels were produced with good spatial and temporal control, with initiator strands acting as nucleation seeds.

In both DNA-polymer and all-DNA gels, elastic shear moduli usually range from tens of $\mathrm{Pa}$ to tens of $\mathrm{KPa}$, mainly depending on DNA (cross-link) concentration. Typical values for all-DNA gelation with moduli around $1 \mathrm{KPa}$ are 20 $\mathrm{mg} / \mathrm{ml}$, or $2 \%$ in weight. At fixed concentration and valence, longer interaction 65 strands generally correspond to stronger bonds and higher melting temperature 
but also to lower molar concentration and lower number of cross-links, therefore resulting in softer gels $[25]^{*}$.

The first example of a dual network hydrogel entirely supramolecular was realized through DNA nanostars and a cucurbit[8]uril (CB[8]) host-guest supramolecular system [27]. The two networks exhibited different and complementary mechanical properties, the DNA one stiffer but brittle and that of $\mathrm{CB}[8]$ more viscoelastic but less susceptibile to breakage.

An alternative approach to the creation of DNA-only hydrogels relies on Rolling Circle Amplification (RCA). The isothermal polymerase phi29 is able to elongate a single DNA strand starting from a circular template and a primer; furthermore, by displacing hybridized strands, phi29 can produce very long filaments with controlled, modular sequence (Fig. 11). By initiating RCA in multiple points, it was possible to create a hydrogel of entangled and physically cross-linked strands [28. The hydrogel displayed a hierarchical internal structure, with densely packed, micron-sized DNA structures, woven together by DNA filaments. Furthermore, it also featured an interesting transition between gel and a quasi-liquid state, reversible upon change of hydration. RCAgenerated filaments can be designed to include e.g. intermolecular i-motifs, so to induce pH-dependent cross-linking of long DNA strands into a hydrogel [17] 185 (Fig. 1 1).

DNA-mediated colloidal self-assembly. Also in the case of colloidal particles, hybridization of DNA filaments, tethered to the surface, has become a prominent means to mediate interactions and to assemble ordered and disordered soft materials 29. A major goal is the spatial and temporal control of the structure of colloidal materials, to reversibly tailor their optical and mechanical properties. In this context, kinetics of self-assembly is challenging because micro-particles host a huge number of DNA strands on their surface, which typically makes the process cooperative and traps the system into metastable states. While these can be tailored and exploited to obtain mesoscopic structures [30, it is often desirable to avoid them. Partially self-complementary strands, thermally 
quenched into hairpins, or pairs of mutually complementary sequences bound on the same particle, can act as a self-protection from interactions, which can be activated at will to control sequential assembly [31. In the last few years, two main advances in the design of DNA-coated colloids have been proposed, namely the use of strand exchange to tune the kinetics and the thermodynamics of interactions, and the introduction of DNA strands to emulsion droplets and lipid vesicles as mobile tethers. An example of the former strategy involves a binary mixture of complementary colloids and free single strands, partially complementary to one population [32, 33]. Binding of the two populations hence proceeded via strand displacement reactions, resulting in a rich phase behaviour, with tunable width of the gas-solid coexistence region and re-entrant melting with fine temperature control. Mobile ligands also allow to avoid kinetic traps along the self-assembly pathway, because DNA tethers can freely diffuse and rearrange upon binding. DNA-mediated interaction of liposomes was investigated as a function of temperature [34]. The combination of ligand mobility and liposome deformability gave rise to unexpected behaviour, like negative thermal expansion and variable porosity of the membranes. The two strategies were somewhat combined into the functionalization of silicone oil droplets [35. These were prepared with pairs of DNA strands made inert by partial mutual hybridization; a first population of droplets was only activated by another population of initiator droplets, binding through a strand displacement, which on turn made possible the binding to a third populations. Five generations of such a sequential self-assembly were demonstrated, which could be functional to the creation of responsive 3D assemblies with tunable mechanical properties.

\section{Shedding light on stressed materials: tools for mechanochromism}

Mapping stress distributions within a material bears obvious interest for applications like early detection of microscopic damage, but it can also help better understanding stress transfer and the processes underlying mechanical failure. In the past decade, spectacular advances have been obtained in the 


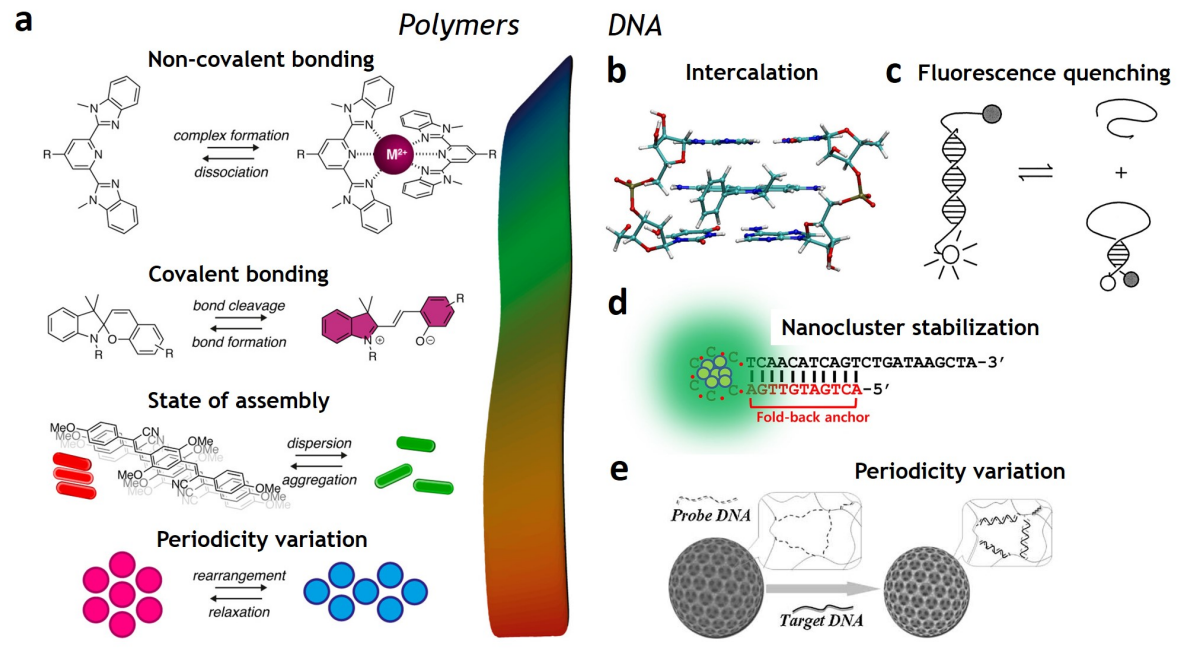

Figure 2: Mechanochromic strategies in polymers and DNA. (a) Examples of mechanisms used in polymers: force-induced rupture of covalent and non-covalent bonds affects the molecular spectral properties of probe molecules linked to the network; deformations induce changes in the aggregation state of dyes or spatial periodicity of photonic crystals, hence modulating fluorescence and structural colors. (b) Ethidium Bromide intercalates in DNA and its fluorescent emission is boosted (credit: K. Langner). (c) In a molecular beacon a fluorescent label is quenched when the hairpin is formed, while opening of the hairpin turns on the emission. (d) A fluorescent complex of DNA and a silver nanocluster is stabilized by stem pairing of a hairpin. (e) Photonic beads made of DNA hydrogel and tagged with quantum dots shrink upon target binding, with consequent shift of their structural color. Reproduced with permission from refs. [2] (Copyright 2017 American Chemical Society), 36] (Copyright 1999 National Academy of Sciences), 37. and 38. 
field of mechanochromic materials, which display an optical response to mechanical stimuli 39]. The optical signal can come from changes in absorption, emission or reflection of light, as the result of variations in the chemical or physical properties of the material and its integrated features. Reflection typically arises from spatially periodic arrangement of nano- or microparticles, or other modulations of dielectric permettivity within the material, with a length scale in the visible range. Shifts in the spacing of such elements affect the structural color. A natural example is chameleon's skin, which features straininduced coloration thanks to nanocrystals embedded in a soft, strain-stiffening matrix. This pattern was recently reproduced through microphase separation in triblock copolymers, with linear chains aggregating in rigid domains and bottlebrush strands forming the matrix [40]. By contrast, modification of the absorption or emission properties is typically associated to structural or conformational variations of suited chromogenic (macro)molecules, or variations of their interactions. By integrating stress-responsive moieties into a matrix, the stress transferred from the macroscale to the molecule or molecular assembly is converted into an optical signal. The main mechanisms for mechanochromism, sketched in Fig. 2a, can be roughly divided into four categories: variations in the periodicity of the aforementioned photonic crystals, rupture of covalent or non-covalent molecular bonds in suited chromophores, changes in the aggrega245 tion state of dyes. As regards the latter, the absorption and/or emission of several dyes can be modified upon aggregation because of electronic interactions between the molecules or as a consequence of restrained conformations [39]. For example, while in typical fluorescent molecules emission is quenched upon aggregation, the class of the so-called aggregation-induced emission fluorophores (like tetraphenylethene) is only fluorescent upon aggregate formation, mostly because of the restriction of intramolecular motion. This enabled a high resolution map of the gelation process of labeled chitosan in $\mathrm{LiOH}-$ urea solutions and clarification of its pathway at high and low temperature [41]. I will give some more details and selected examples about the rupture of covalent and non-covalent bonds, because of their conceptual importance and for the possible 
translation of design strategies (and caveats) into hydrogels and in particular DNA networks. The general principle of mechanochemistry is that suitably designed molecules or molecular complexes can change their absorption and/or emission spectrum upon application of a mechanical stress or strain, so that molecules can be used as force sensors or detectors of bond breaking.

Covalent bonds. Spiropyran (SP) is the first and most widely characterized mechanochromic molecule. Colorless at rest, upon application of a force of few hundreds pN, SP reversibly reacts into merocyanine, which is strongly colored because of its absorption and fluorescent emission properties [42]. Inserted along a polymeric chain or as cross-linker, it is therefore an extremely powerful tool to map stress distribution in a variety of materials. For example, in a stretched polymer network, micro-phase-separated into soft and hard domains, anisotropy of fluorescence polarization provided information on the orientation and degree of alignment of the strained polymers in each phase [43]. Several SP derivatives have been developed and characterized also in terms of forcerate relationships, critical for rational design of mechanochemical response [44]. Indeed, the quantitative assessment of local stresses depends on both the environment and the deformation mode and speed; furthermore, if the relaxation processes occurring in the material are faster than the molecular force response, ${ }_{275}$ a reliable estimate of SP activation time is hampered. As a further proof of the non-trivial translation of optical signal into quantitative stress estimate, mechanical activation of SP was also observed in creep tests, at stress levels below the yield stress of the polymer matrix [45. As the imposed stress was increased, the time required for the onset of SP activation decreased and the onset of the activation always corresponded to a maximum in the strain rate. This seemingly suggests that mechanochemical activity requires stress-enhanced chain mobility and large-scale polymer rearrangements. A current limitation is that activation of SP-like molecules typically occurs only at large macroscopic strain of the material, often corresponding to irreversible plastic deformations, although novel design strategies recently resulted in fluorescence activation at 
around $14 \%$ strain [46].

The other big players in the field are 1,2-dioxetane derivatives, whose loadaccelerated cleavage generates chemiluminescence [47]. In principle, luminescence offers an important advantage over fluorescence because the signal is transient rather than additive, yielding higher contrast and greater spatial and temporal resolution of bond-breaking events. However, emission is very weak, and dioxetane is often coupled to an acceptor fluorescent molecule to boost the quantum yield. In Section 4 I will get back to this molecule for a description of its promising use in the investigation of failure in soft materials.

Supramolecular bonds. Rupture of covalent bonds requires relatively high forces. In soft, water-based biological systems, instead, mechanotransduction is often triggered by much smaller forces determined by changes in supramolecular interactions or protein conformational changes [13, 2]. Therefore dissociation of non-covalent interactions is being increasingly exploited for the realization of mechanochromic materials [39]. An early, protein-based force nanosensor [48] was based on the complex of eYFP and eGFP, both variants of the Green Fluorescent Protein (GFP), with their fluorescent spectra engineered to form a donor/acceptor pair for fluorescence resonance energy transfer (FRET), which sensitively depends on distance. When the pair was held in close proximity by covalently linking both proteins in the cavities of a third protein, and the whole complex was connected to a cross-linked polymer, it sensitively reported strain changes of the matrix through modulation of the FRET signal. More recently, eYFP was inserted at the interface between glass fibers and a polymer matrix in a fiber-reinforced composite. Fiber fracture or detachment from the matrix caused unfolding of the protein and consequent loss of fluorescence, acting as damage reporter 49].

Another interesting class of mechanosensitive supramolecular interactions is represented by metal-ligand complexes, upon which metallosupramolecular polymer gels are formed. Europium can act both as reversible cross-link for telechelic polymers and as photoluminescent reporter of the same complex for- 
mation [50]. Similarly, the various stages of a shape-memory hydrogel were actuated, monitored and quantified through the formation of dynamic, emitting complexes of Europium and alginate [51 $]^{*}$. The diffusion of Europium ions through the hydrogel and the spatially non-homogeneous formation of crosslinks were monitored over time and correlated with the shape change. The process could be reversed by a fluoride solution, which sequestered Europium and effectively removed the cross-links. Modeling of the bending curvature as a function of the number of formed cross-links (directly inferred from the photoluminescent signal) agreed rather well with the experimental data.

A novel, very promising mechanochromic approach was recently proposed and experimentally demonstrated to obtain, rather than a on/off binary signal, a grayscale force detection with sub-pN resolution [52. This was achieved through a conjugated polymer with a semiconducting backbone acting as distributed donor and doped with acceptor molecules along the chain. Upon stretching the chain from a coiled to an elongated conformation, the local monomer density was reduced and fluorescence emission spectrum significantly shifted.

\subsection{DNA-based optical switches}

The DNA dynamic motifs illustrated in Section 2.1 when coupled to suitable chromophores, are also able to modulate an optical signal upon conformational changes or binding events. Most of such strategies have been developed and demonstrated within the framework of biosensing [53] or to monitor selfassembling processes in DNA nanostructures [9], but they are increasingly translated into mechanosensitive structures, like tools for molecular force imaging in cell adhesion studies [54]. The almost exact correspondence between DNAbased optical switches and mechanochromic mechanisms (with the important exception of covalent bond scission) can be appreciated by looking at Fig. 2 The planar, hydrophobic Ethidium Bromide (Fig. 2b) is a prototypical DNA intercalator, whose fluorescence is strongly increased when in the hydrophobic environment of the paired duplex as compared with the free solution state and can therefore act as a reporter of the hybridization. However, pairs of fluores- 
cent labels undergoing FRET, or fluorophore/quencher pairs covalently linked to specific nucleotides are better suited to monitor DNA conformational changes and interactions, like the $\mathrm{pH}$-triggered dissolution of a hydrogel [55].

One of the most widely used motifs is the molecular beacon [36 (Fig. 22), a DNA hydrogel, a photosensitive base analog was inserted in the sticky ends, which reversibly and specifically photo-cross-linked with or cleaved from an adjacent thyimine base upon UV irradiation at two different wavelengths. As a result, reversible transition between hydrogel and liquid solution could be trig375 gered [58.

Fluorescence emission is quenched when the hairpin is closed, while it is turned on upon opening of the hairpin, which results from binding of the loop to a second strand or to some other molecule (if the loop sequence is an aptamer). I will provide in Section 4 examples of the role of this optical switch in the tuning and monitoring of mechanical transitions.

Cytosine-rich sequences can be selected which stabilize silver nanoclusters, hence triggering their fluorescent emission [37] (Fig. 22). Such DNA-silver complex can be inserted into a stable hairpin structure, which can in turn be opened by a competing sequence. In DNA-based photonic crystals [56, 38, (Fig. 2e), stiffness and length of cross-linkers can be modified by binding of target strands or small molecules to single-stranded cross-linkers - an evergreen of DNA nanotechnology -, thereby changing the typical spacing of embedded nanoparticles or quantum dots and hence the structural color.

Although I am mainly focusing on mechanical transitions generating an optical signal, the reverse phenomenon, light-triggered mechanical response, is also a wide and active field of research. For example, DNA strands can be modified with azobenzene molecules, which undergo light-induced, reversible isomerization and thus allow or disfavour hybridization. Acrylamide hydrogels grafted with such DNA strands and their complementary sequences swelled or shrank upon irradiation of UV and visible light, respectively [57. In a nanostar-based jacent thyimine base upon UV irradiation at two different wavelengths. As a .58]. 


\section{Blaze of glory: optical mapping of dissolution and mechanical fail- ure in soft materials}

Soft materials, by definition, can deform significantly when subjected to stress and because they are often characterized by structures at multiple length

scales, their response is typically non-homogeneous. Indeed, several complex fluids, including polymer networks, biopolymer solutions, hydrogels, colloidal gels and microgels, display a variety of intriguing phenomena upon mechanical perturbation, including delayed yielding and non-affine deformation profiles 61], layer fracturing [6], shear-induced changes of their microstructures [62], with a wealth of open questions about their mechanisms of yielding and recovery. Macroscopic rheological approaches can miss important features as heterogeneities or localized stresses are present throughout the sample [63], requiring complementary structural and dynamic information from other techniques. Light and X-ray scattering techniques [64, 65] can access multiple length scales but are typically limited in terms of time and space resolution. Optical imaging [66] is often the most natural choice, although blind to phenomena occurring at the nanoscale. In particular, the in situ combination of rheology and microscopy 62, 6] and microrheology [67, 23], which measures viscoelastic properties on a local scale, can help to bridge macroscopic and microscopic behaviours. Adding spatial and temporal mapping of stress propagation through bond formation and disruption can be extremely beneficial, in particular for those materials like polymer networks and hydrogels - whose building blocks are not individually resolvable. Indeed, mechanochromic molecules can provide such optical mapping.

In the last few years, the most advanced steps in this directions have been obtained with dioxetane cross-links [4] in polymeric networks under stress, upon stretching or swelling. To increase fracture toughness in elastomers while preserving reversibility of deformation, prestretched bonds can be introduced, which first fail when stress is applied, a typical strategy also adopted in hydrogels [1. Dioxetane cross-links allowed to quantify the energy dissipated by 
a

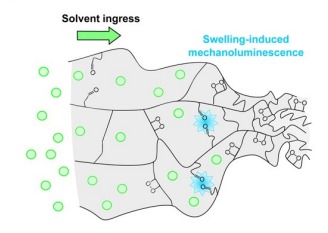

d

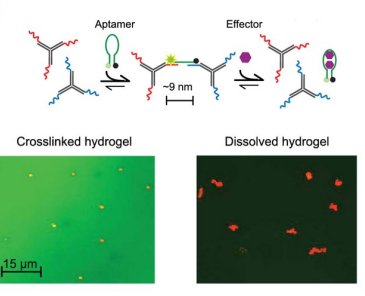

b

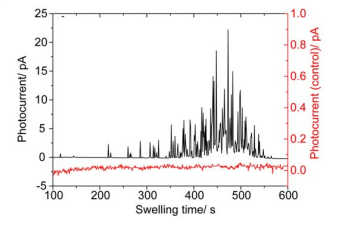

Time- and space-resolved fracture detection

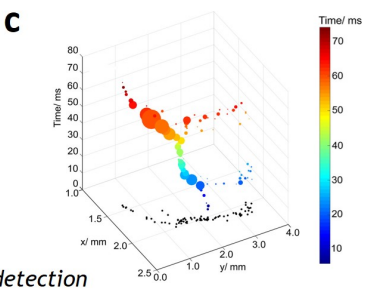

e

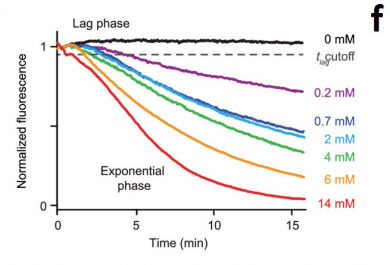

Increase of tracer mobility

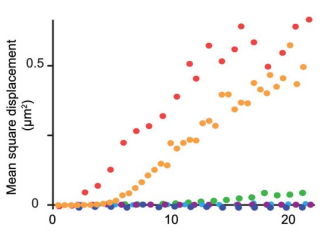

Figure 3: Optical investigation of failure and fluidization. (a) During swelling of a polymer network, broken cross-links emit light. (b) Intermittent and correlated bursts reflect the underlying, non-homogeneous mechanical process, as compared with a stable control sample (red). (c) Propagation and branching of a fracture. The color of the spots depends on the time elapsed from the start of the fracture, their size on the intensity of each measured emission event. Reproduced with permission from [59 ${ }^{* *}$. (d) A DNA nanostar hydrogel is cross-linked through a labelled aptamer sequence; upon addition of its target molecule, the aptamer folds and detaches from the nanostars, which quenches the fluorescent label and induces the gel dissolution. The transition is simultaneously monitored through fluorescence and the mobility of tracer particles dispersed in the gel. (e) Decrease of fluorescence over time for various target concentrations. (f) Increase of tracers mobility at different target concentrations. Same color codes as in e. Reproduced with permission from [60 $]^{* *}$ (Copyright 2017 American Chemical Society). 
such sacrificial bonds and to spatially map bond breakage during a fracture 68. An alternative toughening strategy consists of adding nanoparticles as fillers, which however gives rise to complex mechanical behaviour, like historydependent stress-softening. By optical mapping of the elastomer during repeated tensile cycles at increasing strain amplitude, it was demonstrated that the breakage of a tiny fraction of covalent cross-links between nanofillers and polymer matrix gave a major contribution to stress-softening [69]. Furthermore, the history-dependence of optical emission, associated to covalent bond scission, showed that the stress was mainly oriented in the direction of the macroscopic, uniaxial deformation. However, the observation of further bond breaking upon macroscopic unloading suggested heterogeneity of the local stress distribution, although on a scale below the spatial resolution. Instead, in a cross-linked glassy polymer network undergoing solvent uptake and swelling, it was possible to obtain a spatial and temporal map of localized cascades of light emission from bond-breaking events [59 ${ }^{* *}$ (Fig. 3a). Swelling, associated to a high, nonhomogeneously distributed osmotic stress, gave rise to a series of intermittent bursts with wide variation of intensities and strong temporal clustering (Fig. 3p). Spatial and temporal distribution of the events was obtained in an imaging setup. The smallest and weakest detectable events, lasting few milliseconds, were estimated to correspond to the breakage and emission of about 100 bonds distributed over an area of $\approx 40 \times 40 \mu \mathrm{m}^{2}$, a small fraction of the dioxetane cross-linker molecules. Fig. 35 shows the evolution of a longer fracture event, which propagated at around $30 \mathrm{~mm} / \mathrm{sec}$ and split into branches. Analysis of $2 \mathrm{D}$ spatial and temporal distribution of events, both at short and longer times, supported the notion that the formation of a crack in the sample promoted further cracks in the surrounding material, possibly by the creation of new surface area leading to additional solvent intake. Interestingly, cracks started in the glassy, unswollen part of the network, but with significant localized yielding. This last example clearly shows the wealth of information potentially disclosed by such methods, which may benefit from further quantitative analysis tools like "dynamic activity maps" developed for other soft systems 64. Moreover, similar 
approaches may be extended to the investigation of even softer materials, e.g. complementing microrheology of yielding DNA networks [23].

\subsection{DNA hydrogels}

Indeed, in DNA-based materials much less progress has been obtained, so far, in the optical readout of stress-induced yielding and mechanical transitions. Biosensing hydrogels with combined rheological and fluorescent response to binding events or $\mathrm{pH}$ changes have been reported [55, but with only qualitative correspondence between molecular and mesoscopic scales. A promising step toward a quantitative connection is demonstrated in a hydrogel of DNA nanostars bound through a linker strand, which can also fold into an aptamer for adenosine. Introduction of the target molecule triggered its binding with the DNA strand, stripping it off the nanostars [60 ${ }^{* *}$ (Fig. 3 $\mathrm{d}$ ). By labeling the terminals of the linker strand with a FRET pair, this process was monitored through the decreasing fluorescence signal (Fig. 3p). Binding also caused the disruption of gel connectivity and its dissolution, which was assessed by measuring the mobility of dispersed microparticles through tracking (Fig. 3:). Both processes displayed roughly biphasic kinetics, a lag phase with slow initial change followed by a nearly-exponential decay. Tuning the length of the cross-links changed the kinetics of both responses by orders of magnitude [70. Unbinding, occurring at the molecular scale, proceeded much faster than gel dissolution probed at the microscale, and it displayed different dependence on target concentration, which is not yet entirely understood. However, the fluorescent signal provided direct access to the fraction of connected nanostars at each time (which was found to be spatially homogeneous at all resolvable scales), an information typically missing in microrheological investigations of forming/dissolving hydrogels [67, 23. This allowed to locate the transition from gel to liquid, below a percolation threshold, at about $50 \%$ of intact cross-links, in agreement with theoretical predictions. This kind of approaches, possibly complemented by quantitative analysis of beads motion [66 may be profitably extended to shear-induced fluidization of hydrogels. Finally, further improve- 
ment may come from different reporters, like fluorescent base analogues or pairs of base surrogates which undergo a distinct change from green to red emission upon hybridization to monitor switching states [71.

470

\section{Conclusion and perspective}

I offered a necessarily concise and biased overview of the main elements to create transient networks and soft, mainly DNA-based materials with triggerable mechanical transitions. Given the importance of heterogeneous structure and stress propagation in such behaviour, I focused on the most advanced strategies signal, to create maps of stress/strain with temporal and spatial resolution. I tried to identify analogies and possible contaminations between polymers, hydrogels and DNA-based structures.

In conclusion, I outline a few promising design elements exploited for mechanochromism

480 in polymeric materials and, to my knowledge, not yet demonstrated in DNA hydrogels. As sketched in Fig. 4, I put them in correspondence to existing nucleic acids motifs which may be exploited as mechanical switches, mechanophores and optical reporters.

\subsection{Light it up: fluorescent aptamers}

In section 3 I have described the formation of gels via supramolecular interactions, such as metal complexes acting both as cross-linkers and reporters (Fig. 4a). A similar approach may be replicated in nucleotide-based hydrogels thanks to aptamers based on RNA. From a nanotechnology point of view, RNA shares several of its structural and functional features with DNA, but has a much higher propensity to fold into 3D motifs and is therefore more versatile for the creation of aptamers. Inspired by GFP, Spinach is an 84-bases-long RNA sequence evolved and selected for its ability to activate a fluorescent signal when complexed with a small molecule (DFHBI), which is a mimic of the chromophore of GFP. Spinach has been used in a variety of molecular and cell bi- 


\section{Supramolecular interactions}

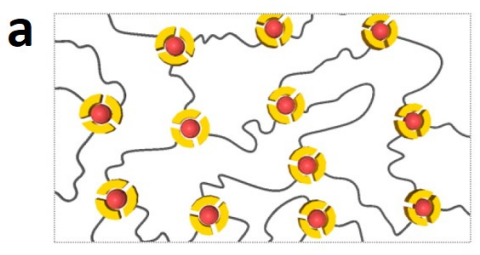

C

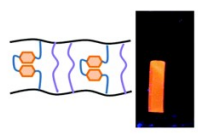

Swelling Swelling $\overleftarrow{\text { Shrinking }}$

$\Pi$

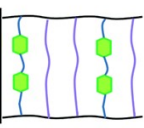

\section{e}

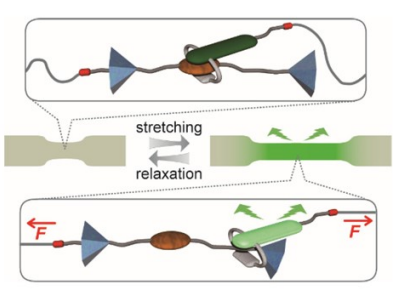

\section{Modular folding}

d

Rotaxanes
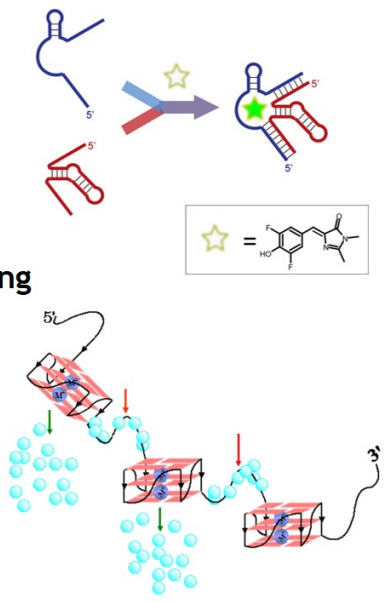

f

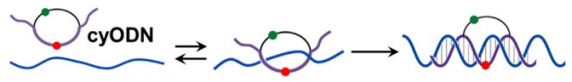

Figure 4: Proposed analogies between mechanochromic strategies and nucleic acids motifs. (a) Sketch of a metallosupramolecular network coordinated by Europium ions. (b) A split Spinach aptamer only emits when the two fragments co-assemble with the DFHBI molecule. (c) Foldamers incorporated as cross-linkers change their emission upon expansion or shrinkage of the gel. (d) Repeated G-quadruplex structures folded along a telomeric DNA. (e) Design for rotaxane-based mechanophore. The interlocked assembly contains a fluorophore-carrying cycle and a quencher. In absence of stress, the fluorophore is quenched, while emission is turned on upon strain application. (f) A DNA pseudorotaxane forms through splippage of a circular filament along a linear one. Reproduced with permission from refs. 50] (Copyright 2014 American Chemical Society), 72 (Copyright 2015 American Chemical Society), 73, 74] (Copyright 2012 American Chemical Society), 75], 76] (Oxford University Press). 
(Mango, Broccoli, ...) have been introduced [77]. Splitting of Spinach into two non-functional fragments, which only restored their activity when co-assembled with DFHBI, enabled an additional set of applications, including the monitoring of dynamic self-assembly processes [72] (Fig. 4b). Furthermore, a split Broccoli aptamer has been demonstrated as a reporter of the folding of a nanoribbon based on RNA origami [78. Fluorescent aptamers may be incorporated into networks as reversible cross-links and very sensitive reporters. Indeed, the force required to mechanically unfold a riboswitch aptamer was estimated to be 10$30 \mathrm{pN}$ [79, in the typical range for DNA and RNA unzipping and one order of magnitude lower than characteristic values for spiropyran [44. More in general, it would be possible to tune the threshold forces by choosing proper DNA nanostructures like Holliday junctions, whose unfolding requires around $50 \mathrm{pN}$ [80. Because of the instability and the high cost of RNA, however, developing a DNA version of a fluorescent aptamer would be a cheaper and more robust alternative, as well as more easily integrated into DNA hydrogels.

\subsection{Fold it: foldamers}

In polymers, the simple design of reversibly unfolding modules, somewhat inspired by titin domains, allows to combine high tensile strength and high extensibility 7 . Reversible loops along the chains may also enable the creation of gels responsive to temperature, external forces or other stimuli [81. Also in the framework of optical reporters, folding can bear some advantages over disruption of bonds. Foldamers are constituted by a pair of rigid chromophores connected by a flexible chain and inserted along the polymer (Fig. 44). They tend to stack, and their forced unfolding generates modifications in absorption and fluorescence emission spectra, with high reversibility and fatigue resistance. A lipogel was produced, based on perylene foldamers as cross-linkers, with a distinct color change from orange to green upon stretching $[73]^{*}$. This allowed to monitor over time the swelling of the gel in an organic solvent, which proceeded from the edges towards the center as the solvent diffused through the 
not performed in the work, would certainly provide useful information on stress propagation.

Translating the loop as folding and reporting element into DNA polymers seems natural, because hairpins and molecular beacons constitute a large fraction of conformational switches and reporters. Furthermore, long tracts with repeating sequences are very frequent in natural DNA strands, in particular in telomeres, where intramolecular G-quadruplexes can form [74] (Fig. 44).

Finally, I note that opening of DNA loops may serve the additional purpose of making available new interactions, previously hidden, within the framwork of the so-called cryptic sites operating in some protein fibers. This design motif, currently explored mostly in surface applications, can introduce strain-stiffening and control the kinetics of relaxation [3].

\subsection{Through the eye of the needle: rotaxanes}

Several mechanochemical schemes involve the force-induced reduction of a chemical activation barrier, but the same reaction can occur upon exposure to light or heat, hence making the response unspecific. Moreover, most mechanically triggered chain scission reactions are irreversible. To overcome these two limits, rotaxane-based mechanoluminophores have been proposed [75]. They consisted of an interlocked assembly containing a fluorophore-carrying cycle, a quencher and two stoppers. The whole complex was integrated into a polymer chain. While at rest, the cycle was on average close to the quencher and the fluorescence off; force-induced displacement of the two elements turned fluorescence on in a reversible way (Fig. 4 4 ). This element produced an easily detectable optical signal that correlated with the applied force, and the design seems easily extendable to other interlocked systems and different chromophores. Among the circular DNA structures possibly functional to the replication within a DNA systems, pseudorotaxanes are based on cyclized oligonucleotides interlocked with single and double strands [76] (Fig. 4f). The thermodynamics and kinetics of their formation via slippage on a DNA strand have been thoroughly investigated. 
As I documented throughout the text, crosstalk and analogies abound between polymer and DNA-based networks. While polymers are often the source of inspiration, the richness of natural DNA motifs and the high versatility of DNA sequence-controlled structures and interactions is allowing to explore solutions which may be mimicked in other polymeric or colloidal system. Morover, given the convergence of recent amazing advances in mechanochromic approaches, in DNA nanotechnology and in tools for quantitative microscopy, a lot more will come in particular as regards stress propagation and fluidization in soft materials. A bright future is ahead of us.

\section{Acknowledgements}

The author acknowledges the long and fruitful collaboration with Tommaso Bellini, and thanks Veronique Trappe, Roberto Cerbino and Fabio Giavazzi for useful discussions. Nicoletta Gnan is gratefully credited for the artwork of the graphical abstract. This work was supported by the Department of Medical Biotechnology and Translational Medicine of the University of Milano (Piano sviluppo Unimi 2017).

\section{References}

[1] L. Montero De Espinosa, W. Meesorn, D. Moatsou, C. Weder, Bioinspired Polymer Systems with Stimuli-Responsive Mechanical Properties (2017). doi:10.1021/acs.chemrev.7b00168

[2] K. M. Herbert, S. Schrettl, S. J. Rowan, C. Weder, 50th Anniversary Perspective: Solid-State Multistimuli, Multiresponsive Polymeric Materials (2017). doi:10.1021/acs.macromol.7b01607.

[3] P. Lavalle, F. Boulmedais, P. Schaaf, L. Jierry, Soft-Mechanochemistry: Mechanochemistry Inspired by Nature, Langmuir 32 (29) (2016) 7265-7276. doi:10.1021/acs. langmuir.6b01768. 
[4] T. Hoffmann, K. M. Tych, M. L. Hughes, D. J. Brockwell, L. Dougan, Towards design principles for determining the mechanical stability of proteins (2013). doi:10.1039/c3cp52142g

[5] J. Wu, P. Li, C. Dong, H. Jiang, B. Xue, X. Gao, M. Qin, W. Wang, Bin Chen, Y. Cao, Rationally designed synthetic protein hydrogels with predictable mechanical properties, Nature Communications 9 (1). doi: $10.1038 / \mathrm{s} 41467-018-02917-6$

[6] S. Sathaye, A. Mbi, C. Sonmez, Y. Chen, D. L. Blair, J. P. Schneider, D. J. Pochan, Rheology of peptide- and protein-based physical hydrogels: Are everyday measurements just scratching the surface?, Wiley Interdisciplinary Reviews: Nanomedicine and Nanobiotechnologydoi:10.1002/wnan.1299.

[7] A. M. Kushner, V. Gabuchian, E. G. Johnson, Z. Guan, Biomimetic design of reversibly unfolding cross-linker to enhance mechanical properties of 3D network polymers, Journal of the American Chemical Society 129 (46) (2007) 14110-14111. doi:10.1021/ja0742176.

[8] J. Chung, A. M. Kushner, A. C. Weisman, Z. Guan, Direct correlation of single-molecule properties with bulk mechanical performance for the biomimetic design of polymers, Nature Materials 13 (11) (2014) 1055-1062. doi:10.1038/NMAT4090.

n [9] N. C. Seeman, H. F. Sleiman, DNA nanotechnology (2017). doi:10.1038/ natrevmats.2017.68

[10] Y. Shao, H. Jia, T. Cao, D. Liu, Supramolecular Hydrogels Based on DNA

n Self-Assembly, Accounts of Chemical Research 50 (4) (2017) 659-668. doi: $10.1021 /$ acs . accounts . 6b00524.

[11] C. Creton, 50th Anniversary Perspective: Networks and Gels: Soft but Dynamic and Tough (2017). doi:10.1021/acs.macromol.7b01698 
[12] L. Imbernon, S. Norvez, L. Leibler, Stress Relaxation and Self-Adhesion of Rubbers with Exchangeable Links, Macromolecules 49 (6) (2016) 21722178. doi:10.1021/acs.macromol.5b02751.

[13] E. A. Appel, J. Del Barrio, X. J. Loh, O. A. Scherman, Supramolecular polymeric hydrogels (2012). doi:10.1039/c2cs35264h.

[14] D. C. Lin, B. Yurke, N. A. Langrana, Inducing reversible stiffness changes in DNA-crosslinked gels, Journal of Materials Research 20 (6) (2005) 14561464. doi:10.1557/JMR. 2005.0186

[15] A. Cangialosi, C. K. Yoon, J. Liu, Q. Huang, J. Guo, T. D. Nguyen, D. H. Gracias, R. Schulman, DNA sequencedirected shape change of photopatterned hydrogels via high-degree swelling, Science 357 (6356) (2017) 11261130. doi:10.1126/science.aan3925.

[16] Y. Hu, C. H. Lu, W. Guo, M. A. Aleman-Garcia, J. Ren, I. Willner, A Shape Memory Acrylamide/DNA Hydrogel Exhibiting Switchable Dual pH-Responsiveness, Advanced Functional Materials 25 (44) (2015) 68676874. doi:10.1002/adfm.201503134.

[17] W. Xu, Y. Huang, H. Zhao, P. Li, G. Liu, J. Li, C. Zhu, L. Tian, DNA Hydrogel with Tunable pH-Responsive Properties Produced by Rolling Circle Amplification, Chemistry - A European Journal 23 (72) (2017) 1827618281. doi:10.1002/chem.201704390.

[18] T. Bellini, R. Cerbino, G. Zanchetta, DNA-based soft phases (2012). doi: $10.1007 / 128\left\{\backslash_{-}\right\} 2011\left\{\backslash_{-}\right\} 230$.

[19] J. S. Kahn, Y. Hu, I. Willner, Stimuli-Responsive DNA-Based Hydrogels: From Basic Principles to Applications, Accounts of Chemical Research 50 (4) (2017) 680-690. doi:10.1021/acs .accounts.6b00542

[20] C. Li, P. Chen, Y. Shao, X. Zhou, Y. Wu, Z. Yang, Z. Li, T. Weil, D. Liu, A writable polypeptide-DNA hydrogel with rationally designed 
multi-modification sites, Small 11 (9-10) (2015) 1138-1143. doi:10.1002/ smll.201401906.

[21] S. Biffi, R. Cerbino, G. Nava, F. Bomboi, F. Sciortino, T. Bellini, Equilibrium gels of low-valence DNA nanostars: A colloidal model for strong glass formers, Soft Matterdoi:10.1039/c4sm02144d.

[22] F. Romano, F. Sciortino, Switching bonds in a DNA gel: An all-DNA vitrimer, Physical Review Letters 114 (7). doi:10.1103/PhysRevLett. 114.078104 .

[23] G. Nava, T. Yang, V. Vitali, P. Minzioni, I. Cristiani, F. Bragheri, R. Osellame, L. Bethge, S. Klussmann, E. M. Paraboschi, R. Asselta, T. Bellini, Newtonian to non-newtonian fluid transition of a model transient network, Soft Matterdoi:10.1039/c8sm00373d.

[24] Z. Xing, A. Caciagli, T. Cao, I. Stoev, M. Zupkauskas, T. O'Neill, T. Wenzel, R. Lamboll, D. Liu, E. Eiser, Microrheology of DNA Hydro-

n gels, Proceedings of the National Academy of Sciencesdoi:10.1073/pnas. 1722206115 .

[25] H. Jiang, V. Pan, S. Vivek, E. R. Weeks, Y. Ke, Programmable DNA Hydrogels Assembled from Multidomain DNA Strands, ChemBioChem (2016) 1156-1162doi:10.1002/cbic.201500686

[26] J. Wang, J. Chao, U. Liu, S. Su, L. Wang, E. Huang, I. Willner, C. Fan, 655 Clamped Hybridization Chain Reactions for the Self-Assembly of Patterned DNA Hydrogels, Angewandte Chemie International Edition 56 (2017) 1712175. doi:https://doi.org/10.1002/anie.201610125.

URL https://doi .org/10.1002/anie.201610125

[27] C. Li, M. J. Rowland, Y. Shao, T. Cao, C. Chen, H. Jia, X. Zhou, Z. Yang, O. A. Scherman, D. Liu, Responsive double network hydrogels of interpenetrating DNA and CB[8] host-guest supramolecular systems, Advanced Materials 27 (21) (2015) 3298-3304. doi:10.1002/adma.201501102. 
[28] J. B. Lee, S. Peng, D. Yang, Y. H. Roh, H. Funabashi, N. Park, E. J. Rice, L. Chen, R. Long, M. Wu, D. Luo, A mechanical metamaterial made from a DNA hydrogel, Nature Nanotechnology 7 (12) (2012) 816-820. doi: 10.1038/nnano.2012.211.

[29] M. Gerth, I. K. Voets, Molecular control over colloidal assembly (2017). doi:10.1039/c6cc09985h.

[30] F. M. Hecht, A. R. Bausch, Kinetically guided colloidal structure formation., Proceedings of the National Academy of Sciences of the United States of America 113 (31) (2016) 8577-82. doi:10.1073/pnas.1605114113. URL http://www.ncbi.nlm.nih.gov/pubmed/27444018http://www. pubmedcentral.nih.gov/articlerender.fcgi?artid=PMC4978265

[31] L. Di Michele, F. Varrato, J. Kotar, S. H. Nathan, G. Foffi, E. Eiser, Multistep kinetic self-assembly of DNA-coated colloids, Nature Communications 4 (1) (2013) 2007. doi:10.1038/ncomms3007. URL http://www. nature.com/articles/ncomms3007

[32] W. Rogers, V. Manoharan, Programming colloidal phase transitions with 1. DNA strand displacement, Science 347 (6222) (2015) 639-642. doi:10. $1126 /$ science. 1259762 .

URL http://science.sciencemag.org/content/347/6222/639

[33] E. W. Gehrels, W. B. Rogers, V. N. Manoharan, Using DNA strand displacement to control interactions in DNA-grafted colloids, Soft Matter 14 (6) (2018) 969-984. doi:10.1039/c7sm01722g.

685 [34] L. Parolini, B. M. Mognetti, J. Kotar, E. Eiser, P. Cicuta, L. Di Michele, Volume and porosity thermal regulation in lipid mesophases by coupling

घ mobile ligands to soft membranes, Nature Communications 6. doi:10. 1038/ncomms6948.

[35] Y. Zhang, A. McMullen, L. L. Pontani, X. He, R. Sha, N. C. Seeman, J. Brujic, P. M. Chaikin, Sequential self-assembly of DNA func- 
tionalized droplets, Nature Communications 8 (1). doi:10.1038/ s41467-017-00070-0.

[36] G. Bonnet, S. Tyagi, A. Libchaber, F. R. Kramer, Thermodynamic basis of the enhanced specificity of structured DNA probes, Proceedings of the National Academy of Sciences 96 (11) (1999) 6171-6176. doi:10.1073/ pnas.96.11.6171.

URL http://www .pnas.org/cgi/doi/10.1073/pnas.96.11.6171

[37] P. Shah, S. W. Choi, H. J. Kim, S. K. Cho, Y. J. Bhang, M. Y. Ryu, P. W. Thulstrup, M. J. Bjerrum, S. W. Yang, Locking-to-unlocking system is an efficient strategy to design DNA/silver nanoclusters (AgNCs) probe for human miRNAs, Nucleic Acids Research 44 (6). doi:10.1093/nar/ gkv1377.

[38] Y. Zhao, X. Zhao, B. Tang, W. Xu, J. Li, J. Hu, Z. Gu, Quantum-dottagged bioresponsive hydrogel suspension array for multiplex label-free DNA detection, Advanced Functional Materials 20 (6) (2010) 976-982. doi:10.1002/adfm.200901812.

[39] C. Calvino, L. Neumann, C. Weder, S. Schrettl, Approaches to polymeric mechanochromic materials, Journal of Polymer Science, Part A: Polymer Chemistry 55 (4) (2017) 640-652. doi:10.1002/pola.28445.

[40] M. Vatankhah-Varnosfaderani, A. N. Keith, Y. Cong, H. Liang, M. Rosenthal, M. Sztucki, C. Clair, S. Magonov, D. A. Ivanov, A. V. Dobrynin, S. S. Sheiko, Chameleon-like elastomers with molecularly encoded strainadaptive stiffening and coloration, Science 359 (6383) (2018) 1509-1513. doi:10.1126/science.aar5308.

715 [41] Z. Wang, J. Nie, W. Qin, Q. Hu, B. Z. Tang, Gelation process visualized by 【 aggregation-induced emission fluorogens, Nature Communications 7. doi: $10.1038 /$ ncomms 12033 
[42] D. A. Davis, A. Hamilton, J. Yang, L. D. Cremar, D. Van Gough, S. L. Potisek, M. T. Ong, P. V. Braun, T. J. Martínez, S. R. White, J. S. Moore, N. R. Sottos, Force-induced activation of covalent bonds in mechanoresponsive polymeric materials, Nature 459 (7243) (2009) 68-72. doi:10.1038/nature07970.

[43] C. K. Lee, B. A. Beiermann, M. N. Silberstein, J. Wang, J. S. Moore, N. R. Sottos, P. V. Braun, Exploiting force sensitive spiropyrans as molecular level probes, Macromolecules 46 (10) (2013) 3746-3752. doi:10.1021/ ma4005428,

[44] G. R. Gossweiler, T. B. Kouznetsova, S. L. Craig, Force-rate characterization of two spiropyran-based molecular force probes, Journal of the American Chemical Society 137 (19) (2015) 6148-6151. doi:10.1021/jacs. $5 \mathrm{~b} 02492$

[45] C. M. Degen, P. A. May, J. S. Moore, S. R. White, N. R. Sottos, Timedependent mechanochemical response of SP-cross-linked PMMA, Macromolecules 46 (22) (2013) 8917-8921. doi:10.1021/ma4018845.

[46] Z. Li, R. Toivola, F. Ding, J. Yang, P. N. Lai, T. Howie, G. Georgeson, S. H. Jang, X. Li, B. D. Flinn, A. K. Jen, Highly Sensitive Built-In Strain Sensors for Polymer Composites: Fluorescence Turn-On Response through Mechanochemical Activation, Advanced Materials (2016) 6592-6597doi: 10.1002/adma.201600589.

[47] Y. Chen, A. J. Spiering, S. Karthikeyan, G. W. Peters, E. W. Meijer, R. P. Sijbesma, Mechanically induced chemiluminescence from polymers incorporating a 1,2-dioxetane unit in the main chain, Nature Chemistry 4 (7) (2012) 559-562. doi:10.1038/nchem.1358

[48] N. Bruns, K. Pustelny, L. M. Bergeron, T. A. Whitehead, D. S. Clark, Mechanical nanosensor based on fret within a thermosome: Damage-reporting polymeric materials, Angewandte Chemie - International Edition 48 (31) (2009) 5666-5669. doi:10.1002/anie.200900554. 
[49] O. Rifaie-Graham, E. A. Apebende, L. K. Bast, N. Bruns, Self-Reporting Fiber-Reinforced Composites That Mimic the Ability of Biological Materials to Sense and Report Damage, Advanced Materials 30 (19). doi: 10.1002/adma.201705483.

[50] D. W. Balkenende, S. Coulibaly, S. Balog, Y. C. Simon, G. L. Fiore, C. Weder, Mechanochemistry with metallosupramolecular polymers, Journal of the American Chemical Society 136 (29) (2014) 10493-10498. doi: $10.1021 / \mathrm{ja} 5051633$

[51] W. Lu, C. Ma, D. Zhang, X. Le, J. Zhang, Y. Huang, C. F. Huang, T. Chen, Real-Time in Situ Investigation of Supramolecular Shape Memory Process by Fluorescence Switching, Journal of Physical Chemistry C 122 (17) (2018) 9499-9506. doi:10.1021/acs.jpcc.8b01689.

[52] T. van de Laar, H. Schuurman, P. van der Scheer, J. Maarten van Doorn, 760 口 J. van der Gucht, J. Sprakel, Light from Within: Sensing Weak Strains and FemtoNewton Forces in Single Molecules, Chem 4 (2) (2018) 269-284. doi:10.1016/J.CHEMPR.2017.12.016, URL https://www.sciencedirect.com/science/article/pii/ S2451929417305181

[53] G. Zanchetta, R. Lanfranco, F. Giavazzi, T. Bellini, M. Buscaglia, Emergघ ing applications of label-free optical biosensors (2017). doi:10.1515/ nanoph-2016-0158.

[54] Y. Zhang, C. Ge, C. Zhu, K. Salaita, DNA-based digital tension probes reveal integrin forces during early cell adhesion, Nature Communications 5 (1) (2014) 5167. doi:10.1038/ncomms6167.

URL http://www .nature.com/articles/ncomms6167

[55] S. Lu, S. Wang, J. Zhao, J. Sun, X. Yang, A pH-controlled bidirectionally pure DNA hydrogel: Reversible self-assembly and fluorescence monitoring, Chemical Communicationsdoi:10.1039/c8cc01603h. 
[63] D. T. N. Chen, Q. Wen, P. Janmey, J. Crocker, A. G. Yodh, Rheology of soft materials, Annual Review of Condensed Matresponse based on aptamer functionalized colloidal crystal hydrogels, Nanoscale 7 (17) (2015) 7565-7568. doi:10.1039/c5nr00586h.

[57] L. Peng, M. You, Q. Yuan, C. Wu, D. Han, Y. Chen, Z. Zhong, J. Xue, W. Tan, Macroscopic volume change of dynamic hydrogels induced by reversible DNA hybridization, Journal of the American Chemical Society 134 (29) (2012) 12302-12307. doi:10.1021/ja305109n.

[58] D. Kandatsu, K. Cervantes-Salguero, I. Kawamata, S. Hamada, S. i. M. Nomura, K. Fujimoto, S. Murata, Reversible GelSol Transition of a Photo-Responsive DNA Gel, ChemBioChem (2016) 1118-1121doi:10. 1002/cbic.201600088,

[59] J. M. Clough, J. Van Der Gucht, R. P. Sijbesma, Mechanoluminescent Imaging of Osmotic Stress-Induced Damage in a Glassy Polymer Network,

1. Macromolecules 50 (5) (2017) 2043-2053. doi:10.1021/acs.macromol. $6 \mathrm{~b} 02540$

[60] A. J. Simon, L. T. Walls-Smith, M. J. Freddi, F. Y. Fong, V. Gubala, K. W. Plaxco, Simultaneous Measurement of the Dissolution Kinetics of Responsive DNA Hydrogels at Multiple Length Scales, ACS Nano 11 (1) (2017) 461-468. doi:10.1021/acsnano.6b06414

[61] T. Divoux, C. Barentin, S. Manneville, From stress-induced fluidization processes to Herschel-Bulkley behaviour in simple yield stress fluids, Soft Matter 7 (18) (2011) 8409-8418. doi:10.1039/c1sm05607g.

URL http://xlink.rsc.org/?DOI=c1sm05607g

[62] N. Koumakis, E. Moghimi, R. Besseling, W. C. Poon, J. F. Brady, G. Petekidis, Tuning colloidal gels by shear, Soft Matter 11 (23) (2015) 46404648. doi:10.1039/c5sm00411j. 
ter Physics 1 (1) (2010) 301-322. doi:https://doi.org/10.1146/ annurev-conmatphys-070909-104120.

URL wWw . annualreviews . org

[64] A. Duri, D. A. Sessoms, V. Trappe, L. Cipelletti, Resolving long-range spatial correlations in jammed colloidal systems using photon correlation imaging, Physical Review Lettersdoi:10.1103/PhysRevLett.102.085702

[65] G. Zanchetta, R. Cerbino, Exploring soft matter with x-rays: From the discovery of the DNA structure to the challenges of free electron lasers (2010). doi:10.1088/0953-8984/22/32/323102

[66] R. Cerbino, Quantitative optical microscopy of colloids: The legacy of Jean Perrin (2018). doi:10.1016/j.cocis.2018.03.003.

[67] K. M. Schultz, A. D. Baldwin, K. L. Kiick, E. M. Furst, Measuring the modulus and reverse percolation transition of a degrading hydrogel, ACS Macro Letters 1 (6) (2012) 706-708. doi:10.1021/mz300106y.

[68] E. Ducrot, Y. Chen, M. Bulters, R. P. Sijbesma, C. Creton, Toughening elastomers with sacrificial bonds and watching them break, Science 344 (6180) (2014) 186-189. doi:10.1126/science.1248494.

${ }_{820}^{6}$ [69] J. M. Clough, C. Creton, S. L. Craig, R. P. Sijbesma, Covalent Bond Scission in the Mullins Effect of a Filled Elastomer: Real-Time Visualization with Mechanoluminescence, Advanced Functional Materials 26 (48) (2016) 9063-9074. doi:10.1002/adfm.201602490

[70] A. J. Simon, L. T. Walls-Smith, K. W. Plaxco, Exploiting the 825 conformational-selection mechanism to control the response kinetics of

口 a "smart" DNA hydrogel, Analyst 143 (11) (2018) 2531-2538. doi: $10.1039 / \mathrm{c} 8 \mathrm{an} 00337 \mathrm{~h}$

[71] S. Barrois, H. A. Wagenknecht, In-stem labelling allows visualization of DNA strand displacements by distinct fluorescent colour change, Organic 
and Biomolecular Chemistry 11 (19) (2013) 3085-3088. doi:10.1039/ c3ob40435h.

[72] T. A. Rogers, G. E. Andrews, L. Jaeger, W. W. Grabow, Fluorescent monitoring of RNA assembly and processing using the split-spinach aptamer, ACS Synthetic Biology 4 (2) (2015) 162-166. doi:10.1021/sb5000725.

[73] J. Chen, A. W. Ziegler, B. Zhao, W. Wan, A. D. Li, Chemomechanicalforce-induced folding-unfolding directly controls distinct fluorescence dualcolor switching, Chemical Communications 53 (36) (2017) 4993-4996. doi : $10.1039 / \mathrm{c} 7 \mathrm{cc} 01643 \mathrm{c}$

[74] H. Yu, X. Gu, S. I. Nakano, D. Miyoshi, N. Sugimoto, Beads-on-a-string structure of long telomeric dnas under molecular crowding conditions, Journal of the American Chemical Society 134 (49) (2012) 20060-20069. doi:10.1021/ja305384c.

[75] Y. Sagara, M. Karman, E. Verde-Sesto, K. Matsuo, Y. Kim, N. Tamaoki, C. Weder, Rotaxanes as mechanochromic fluorescent force transducers in polymers, Journal of the American Chemical Society 140 (5) (2018) 15841587. doi:10.1021/jacs.7b12405.

[76] K. Onizuka, T. Chikuni, T. Amemiya, T. Miyashita, K. Onizuka, H. Abe, F. Nagatsugi, Pseudorotaxane formation via the slippage process with chemically cyclized oligonucleotides, Nucleic Acids Research 45 (9) (2017) 5036-5047. doi:10.1093/nar/gkx265.

[77] J. Ouellet, RNA Fluorescence with Light-Up Aptamers, Frontiers in Chemistry 4. doi:10.3389/fchem.2016.00029

a URL http://journal.frontiersin.org/Article/10.3389/fchem. 2016.00029/abstract

[78] E. Torelli, J. W. Kozyra, J. Y. Gu, U. Stimming, L. Piantanida, K. Voïtchovsky, N. Krasnogor, Isothermal folding of a light-up bio- 
orthogonal RNA origami nanoribbon, Scientific Reports 8 (1). doi: $10.1038 / \mathrm{s} 41598-018-25270-6$

[79] P. C. Anthony, C. F. Perez, C. García-García, S. M. Block, Folding energy landscape of the thiamine pyrophosphate riboswitch aptamer, BIOPHYSICS AND COMPUTATIONAL BIOLOGY 109 (5) (2012) 14851489. doi:10.1073/pnas.

[80] P. Shrestha, T. Emura, D. Koirala, Y. Cui, K. Hidaka, W. J. Maximuck, M. Endo, H. Sugiyama, H. Mao, Mechanical properties of DNA origami nanoassemblies are determined by Holliday junction mechanophores, Nucleic Acids Researchdoi:10.1093/nar/gkw610.

[81] S. Biswas, V. V. Yashin, A. C. Balazs, "patterning with loops" to dynamically reconfigure polymer gels, Soft Matter 14 (17) (2018) 3361-3371. doi:10.1039/c8sm00270c. 